\title{
Analysis of Radiation Propagation inside a Hierarchical Solar Volumetric Absorber ${ }^{+}$
}

\author{
Luca Pratticò ${ }^{1,2, *}$, Ruben Bartali ${ }^{2}$, Luigi Crema ${ }^{2}$ and Enrico Sciubba ${ }^{1, *}$ \\ 1 Department of Astronautical, Electric and Energy Engineering, Sapienza University of Rome, \\ 00185 Rome, Italy \\ 2 Fondazione Bruno Kessler, ARES Unit, 38123 Trento, Italy; bartali@fbk.eu (R.B.); crema@fbk.eu (L.C.) \\ * Correspondence: luca.prattico@uniroma1.it (L.P.); enrico.sciubba@uniroma1.it (E.S.) \\ + Presented at the First World Energies Forum, 14 September-5 October 2020; Available online: \\ https://wef.sciforum.net/.
}

Published: 12 September 2020

\begin{abstract}
The solar receiver is a critical component of concentrated solar power technology; it works as a heat exchanger, transforming the concentrated solar radiation into high-temperature heat. Volumetric receiver technologies, using air as a heat transfer fluid, are designed to reach higher temperatures than the current receiver technology, which is limited by material resistance and fluid instability. The higher temperature, up to $1200 \mathrm{~K}$, could be used in high-temperature industrial processes or a high-temperature thermodynamic cycle. A correct radiation propagation is essential to develop their performances, reducing reflection and emission losses and promote the heat transfer to the fluid. In this study, the optical behaviour of a hierarchical volumetric receiver (HVR) developed in Bruno Kessler Foundation (FBK) has been studied using Monte Carlo ray tracing (MCRT) simulations. The simulations have been validated in an experimental setup that evaluates the light transmissivity of the HVR porous structure. Two different HVR structures are evaluated with MCRT simulations that use a real solar dish geometry to configure a complete concentrated solar power (CSP) plant. Results show that frontal and rear losses are, respectively, $12 \%$ and $3 \%$ of the incoming concentrated radiation. Inside the HVR, $15 \%$ of the incoming power is propagated trough the lateral void spaces. Therefore, the power spreading avoids the overconcentration of the centre of the focalized area. The HVR optical behaviour has been investigated, showing an optical efficiency of $85 \%$.
\end{abstract}

Keywords: concentrated solar power; radiation propagation; volumetric receivers; Monte Carlo ray tracing; high-temperature heat

\section{Introduction}

The international agreements, which have been stipulated to mitigate the global warming phenomenon, are aiming to reduce the $\mathrm{CO}_{2}$ emissions and limit the global average temperature increase to $2{ }^{\circ} \mathrm{C}$ [1]. $\mathrm{CO}_{2}$ emissions together with other greenhouse gas (GHG) emissions are widely recognized as the main driver of global warming [2]. The heat production for the industrial sector and for the electricity generation represents more than $40 \%$ of the global GHG emissions [2]. Therefore, the research in carbon-free heat production needs to be developed further. Among renewable energy sources, solar energy has the potential to alleviate the aforementioned energy issues [3]. Concentrated solar power (CSP) technologies use mirrors to concentrate the solar radiation and produce heat. CSP is considered as promising and viable technology capable of replacing fossil fuel consumption for the heat and power production [4]. Point focusing configurations, like central receiver towers or solar dishes, are capable of higher concentration ratios compared to the line focusing configuration [5]. High concentrated radiation, with fluxes over $500 \mathrm{~kW} / \mathrm{m}^{2}$, can produce 
high-temperature heat over $700{ }^{\circ} \mathrm{C}$. This high-temperature heat can be used to efficiently produce electrical power or to feed high-temperature chemical processes (e.g., cement manufacturing, mineral and metallurgical extraction, solid waste gasification) [6], and advanced solar fuel synthesis [7].

A core component of these plants is the solar receiver that is placed in the focal area of the concentrating mirrors. The receiver is essentially a heat exchanger which performs the conversion of concentrated radiation into heat. The state of the art of the point focusing CSP technologies is based on tubular solar receiver that can bear only moderate concentration ratios, using oil, water-steam or molten salt as a heat transfer fluid (HTF). Therefore, the overall efficiency from solar to electricity is below $20 \%$ and the thermochemical routes are beyond reach [7]. According to Carnot's theorem, higher temperatures in the heat source are essential to increase the thermodynamic power cycle efficiency; in CSP the heat source is represented by heat transfer fluid heated in the receiver [8]. The current tubular solar receiver technologies using molten salt as a HTF are not suitable for higher concentrated flux and temperature (over $600^{\circ} \mathrm{C}$ ) due the salt instability and material mechanical resistance [9].

A promising path for the high-temperature receivers is represented by the volumetric type [10]. These receivers use a porous structure that absorbs "volumetrically" the solar radiation [11]. In Figure 1 , a sketch of the working principles of the two receiver technologies is reported, Tubular Receivers (TR) and Volumetric Receivers (VR). On the same side that the absorber is facing, the radiation enters a gaseous HTF, typically air, which exchanges heat by convection cooling the absorber structure [12]. A VR has some advantages if compared with TR. A VR does not have any limitation in temperature coming from the HTF stability. They could achieve the so called "volumetric effect" that is described by Avila et al. [11] as "the temperature on the irradiated side of the absorber to be lower than the outlet temperature". This low temperature in front of the receiver reduces losses of radiative emission to the environment [13]. However only some VR have shown the presence of this effect in particular conditions [14].

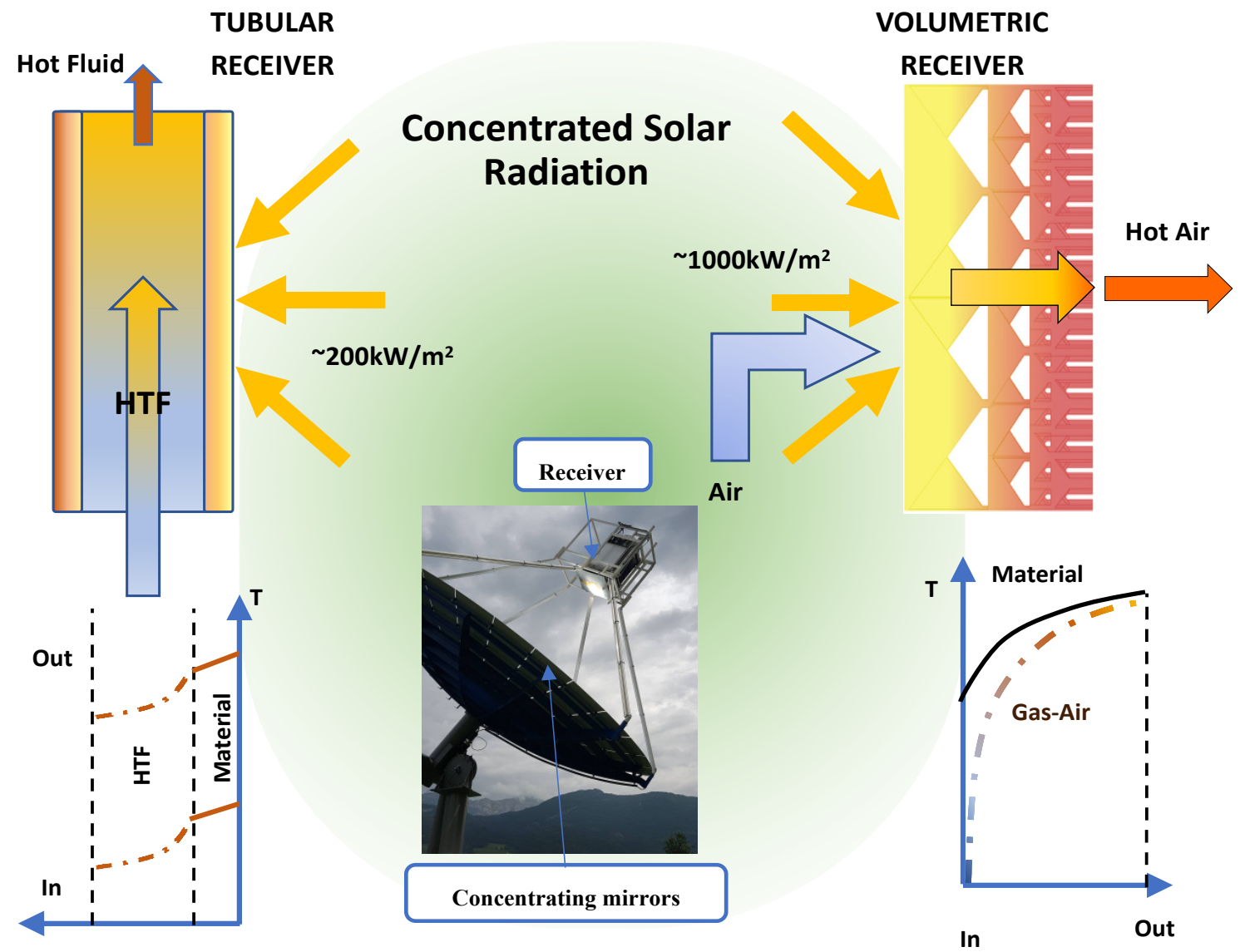

Figure 1. Sketch of receiver working principles: on the left tubular receivers, on the right volumetric technology. 
VR receivers may have ordered or un-ordered structures that form their porous geometries. Both the categories could use ceramic or metallic materials. The first category may be monolithic [15-17], wire meshes [3], or pinned [18]. The unstructured are mainly open-cell foams [13]. Some other authors have proposed packed bed configurations [19]. A hierarchical volumetric receiver (HVR) [20] was proposed to improve the performances of a structured VR by enhancing the volumetric effect [14].

This paper presents a detailed analysis of the radiation propagation inside the hierarchical volumetric absorber using both experimental measurements and numerical simulations. In the first part, the transmissivity of the HVR prototype is estimated experimentally using a solar simulator as a radiation source and a solar power meter as a probe. Considering this setup and the geometry of the prototype, the numerical simulations were conducted using the Monte Carlo ray-tracing technique. The simulation has been validated comparing the simulation results with the experimental ones. In the second part, taking into account the lab results, we modelled the performance of two HVR geometries coupled with a real concentrated solar system. In these simulations, a solar dish geometry of a real prototype [21], Contest project, was used as a concentrated source that hits the HVR geometry.

\section{Methodology}

In this section, the methodology applied in this study will be explained in detail. The methodology is thus divided into: (a) a numerical part focused on the geometrical configuration and boundary conditions of Monte-Carlo ray tracing (MCRT) simulations, (b) an experimental part where the experimental setup designed to validate numerical simulations is described.

\subsection{Numerical Methodology}

Numerical simulations were conducted using the open-source Monte Carlo ray-tracing software Tonatiuh [22]. The HVR was simulated in two different simulation setups. In the first, the radiative flux was transmitted through the receiver volume simulating similar conditions to the experimental setup, configuration 2. In the second, the receiver was simulated for a real CSP system in two different sizes-configuration 1 and configuration 2, see Figure 2. The configuration 1 had the same hierarchical structure of configuration 2 but the receiver frontal area was larger, to allow the adequate distribution of solar power provided by a large parabolic dish, see Figure 2.

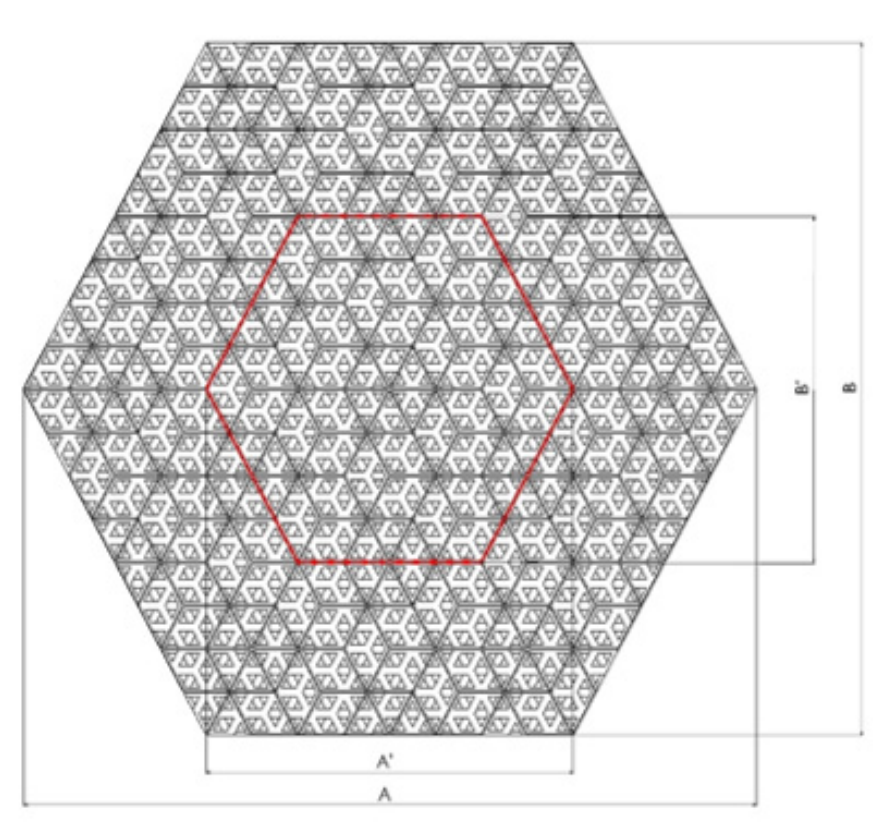

(a)

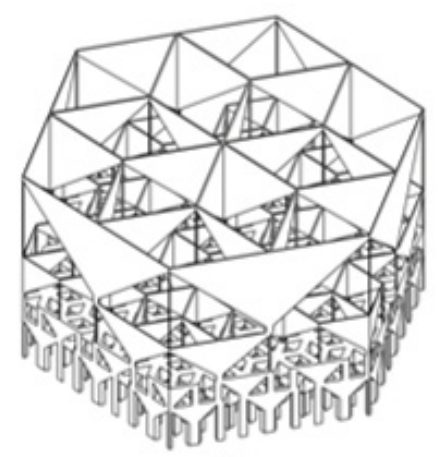

(b)

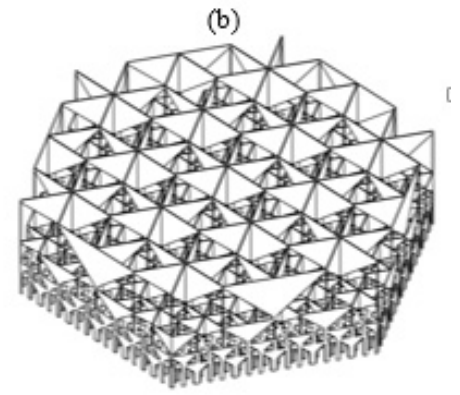

(c)

Figure 2. (a) Frontal view of hierarchal receiver geometries that are numerically simulated; the configuration 1 is denoted by dimension $\mathrm{A}$ and $\mathrm{B}$, configuration 2 is underlined in red. (b) Isometric view of configuration 2. (c) Isometric view of configuration 1. 


\subsubsection{MCRT Collimated Source Simulations}

These simulations were designed to validate our MCRT methodology comparing these simulations with measurements from the experimental setup. Collimated source reproduces the experimental conditions that are illustrated in the Section 2.2. The receiver geometry is represented in Figure 2. The simulation configuration consisted of a collimated radiative source, the HVR geometry and two auxiliary planes. The radiation source reproduced the light coming from the Abet Solar simulator [23], the direct normal irradiation (DNI) was set to $700 \mathrm{~W} / \mathrm{m}^{2}$. The circumsolar ratio of the source was set to $4.65 \mathrm{mrad}$ and the angle $\alpha$ between the radiative source and receiver main axis was $90^{\circ} \mathrm{deg}$. The receiver reflectance was set to $40 \%$ with a non-specular behaviour. The auxiliary planes were two square planar surfaces, with $10 \mathrm{~mm}$ edges, parallel to the frontal surface of the receiver and orthogonal to main axis, represented in Figure 3a. These planes acted as a probe for the incoming radiation; however, the planes did not change the photons' behaviour and trajectories. The first was positioned next to the receiver front face, underlined in Figure 3b. The second, instead, was just behind the rear face. This configuration was designed to investigate the average irradiance on these surfaces measured as the number of incident photons times the photon power divided by the surface area.

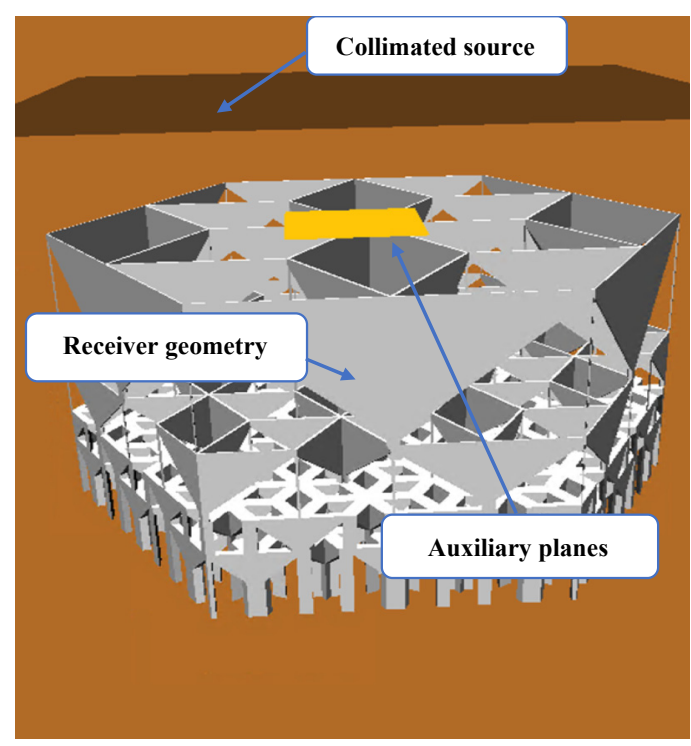

(a)

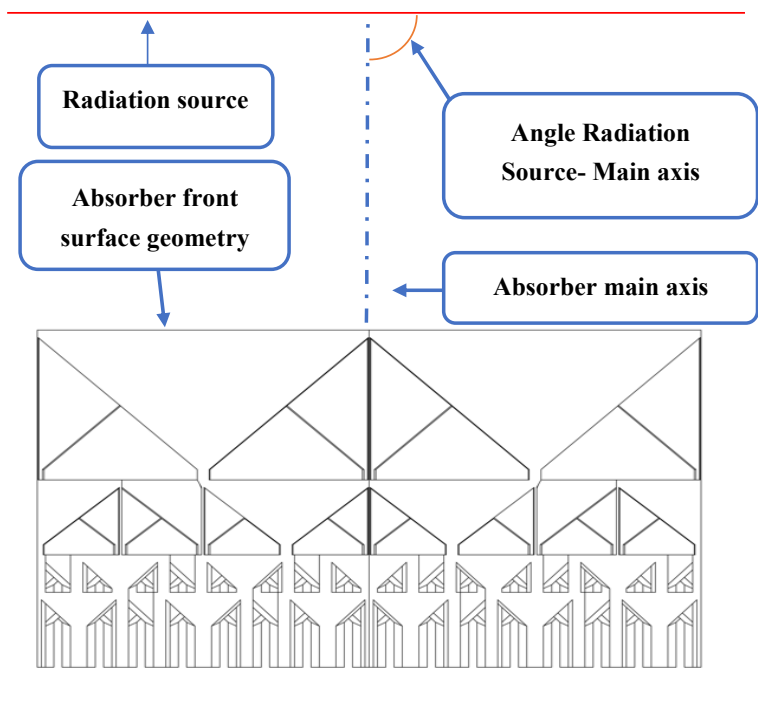

(b)

Figure 3. (a) Configuration of collimated source Monte-Carlo ray tracing (MCRT) simulations: radiative source (above), hierarchical volumetric receiver (HVR) absorber geometry (centre), auxiliary planes (below). (b) Geometrical arrangement in collimated source configuration underlining the angle between radiation source and receiver main axis.

\subsubsection{MCRT Simulations Using Concentrated Radiative Source}

The HVR optical analysis was conducted on two receiver geometries. In the first, a wider system was simulated, representing the absorber cup; in the second, only the central cell, underlined in red in Figure 2a, was under investigation. The bigger hexagon (configuration 1), which represents the receiver frontal area, had a side that was the twice the size of the smaller one (configuration 2). The results of complete cup simulation showed a higher accuracy to represent the real behaviour of the radiation evolution that happens inside the HVR geometry. Indeed, in the first configuration, several cells were simulated, thus it was possible to evaluate the radiation evolution through non-solid lateral surfaces of HVR elementary cells. It has to be noted that this lateral radiation evolution has a great importance in the radiation propagation in the whole receiver. This importance is due to the capability of non-solid surfaces to spread the concentrated radiation. A correct radiation flux spreading through lateral surface is crucial to avoid fluxes that are unbearable for the absorber 
structure. In addition to that, in the configuration 2, this evolution needed to be carefully evaluated to obtain a realistic solution by properly tuning the boundary conditions that represented other parts of the whole absorber geometry (configuration 1). However, in the configuration 1, the computational effort was high, including geometry generation, simulation and post processing (it needed more than two days to be completed).

Both receivers analysed in MCRT simulations use a geometrical configuration that consists in: a solar source, an optical concentrator, hierarchical receiver and auxiliary planes.

The solar source, represented in Figure 4 by a black box, produces up to $10^{8}$ photons with a pillbox sun-shape. The source had an equivalent solar DNI of $0.001 \mathrm{~W} / \mathrm{mm}^{2}$ and circumsolar ratio of $4.65 \mathrm{mrad}$.

The optical concentrator was designed based on the geometrical generatrix of a solar dish of the CONTEST project [21]. The solar dish used was a spherical-parabolical optical concentrator with an aperture diameter of $8.6 \mathrm{~m}$. In the central part of the dish there were spherical curved mirrors with a curvature of $9.293 \mathrm{~m}$. The peripheral ring had, instead, a parabolic curvature, with a focal distance of $4.5 \mathrm{~m}$. As parameters of the mirrors, we set $85 \%$ of reflectivity and a specular error of $4 \mathrm{mrad}$.

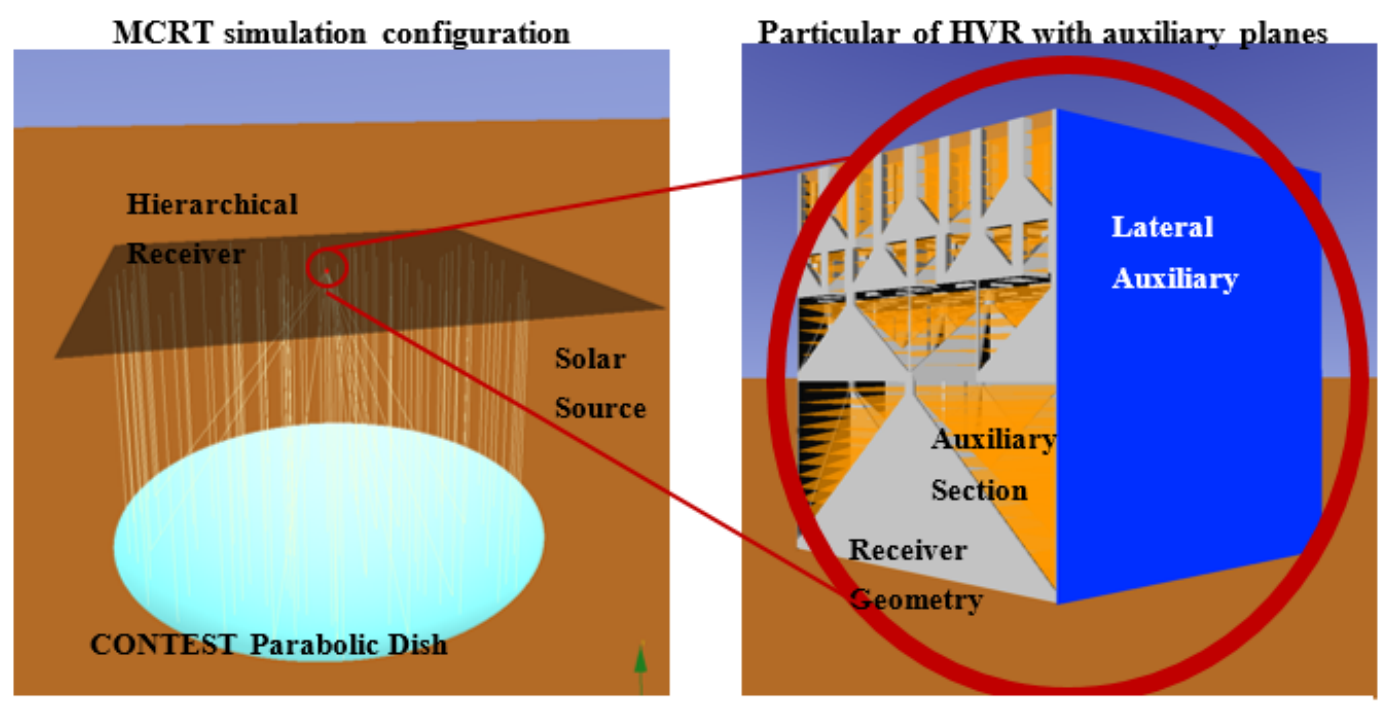

(a)

(b)

Figure 4. MCRT simulation geometrical configuration. (a) Overall configuration: Contest Project solar dish (bottom), HVR receiver geometry (centre) and solar source (top). (b) Particular area of the configuration: lateral auxiliary plane (right), receiver geometry (left grey), auxiliary section planes (left orange).

The HVR geometry is shown in Figure 4 on the right part, located at $4.5 \mathrm{~m}$ from the dish centre (i.e., in dish focal distance). Two types of planes were used as probe and as boundary conditions in MCRT simulations. The so-called auxiliary section planes, represented in orange in Figure 4, were parallel to the frontal face of the receiver. These planes were acting as virtual probes without any interaction with the photon path inside the receiver. Only the last plane, situated at the rear face, was a perfect absorber. The lateral auxiliary planes, represented in blue, were located in the lateral faces of the receiver, transversally to the absorber frontal face. These were intended to represent the insulating material around the receiver, for the configuration 1 . On the other hand, in a small receiver simulation, the lateral planes are boundary conditions that represent the transfer of photons between receiver cells. As aforementioned, due the concentrated radiation distribution, only a part of the radiative power that is coming from the central receiver cells to the lateral ones is coming back. The percentage of this power was evaluated using the absorber in configuration 1, hence in these simulations some additional auxiliary planes were added. These additional planes were located in the same position of lateral auxiliary planes in simulating configuration 2 , which coincides with the limit highlighted in red in Figure 2a. 


\subsection{Experimental Methodology}

Experimental Measurements of Radiation Transmissivity

In this experimental part, the radiation transmitted through the whole HVR structure is measured. The experimental setup, represented in Figure 5, was formed by radiation source, the receiver prototype and radiation meter. The radiation source was the solar simulator "Sun 2000 " by Abet technologies [23] equipped with a $1000 \mathrm{~W}$ xenon lamp and AM 1.5 filter. The solar simulator was capable of a maximum irradiance of $2000 \mathrm{~W} / \mathrm{m}^{2}$ that could be attenuated by a beam attenuator and power adjustment of the xenon lamp. The absorber prototype, with the geometry reported in Figure $2 \mathrm{~b}$, was kept in position by a plexiglass support. The radiation meter model RS PRO Solar Power Meter ISM400 [24] was positioned just below the prototype.

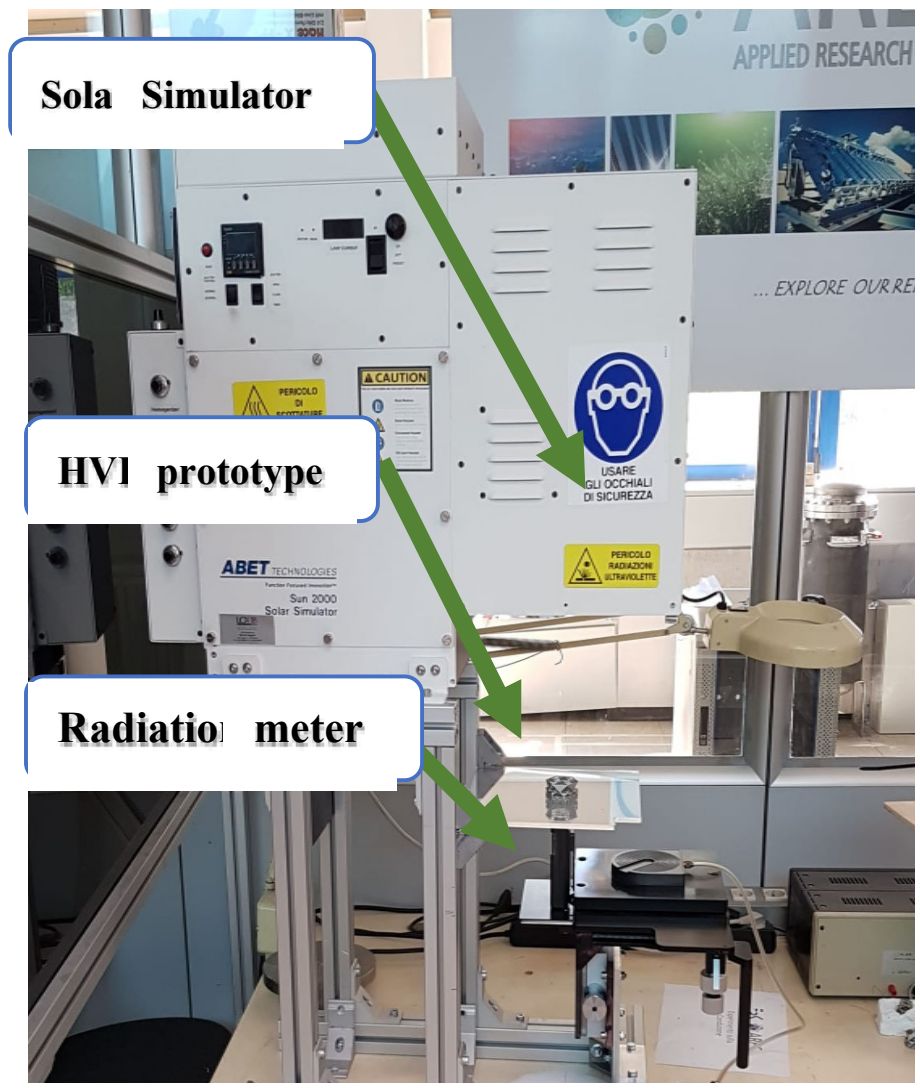

Figure 5. Experimental setup of radiation transmissivity through the HVR geometry: solar simulator (above), HVR prototype (centre), radiation meter (below).

The irradiance measurement has been taken after two hours of solar simulator electronics power stabilization.

\section{Results}

\subsection{Result of Transmissivity Experimental Setup}

In Table 1, the experimental results of irradiance through the HVR absorber under a collimated source are reported. The input irradiance that comes from the solar simulator is reported in the table's first row. The reported value is the average of ten measurements with a value of $365 \mathrm{~W} / \mathrm{m}^{2}$ and a standard deviation of $2 \mathrm{~W} / \mathrm{m}^{2}$. The light transmitted through the receiver geometry was measured ten times in different zones of the receiver. As result the transmissivity had an average irradiance of $33.8 \mathrm{~W} / \mathrm{m}^{2}$ and a standard deviation of $5.6 \mathrm{~W} / \mathrm{m}^{2}$. These transmittance results point out that about a tenth $(9.2 \%)$ of incoming radiative power is lost in the receiver rear face. This high level of transmitted 
power is due to characteristics of impinging radiation, similar to the solar irradiance, that has an aperture angle of only $4.65 \mathrm{mrad}$, far lower than the designed aperture angle for the HVR that is $60^{\circ}$.

Table 1. Result of radiative transmissivity experimental setup.

\begin{tabular}{ccc}
\hline Zone & \multicolumn{2}{c}{ Irradiance W/m } \\
\hline Without Receiver + STDEV & $365 \pm 2$ & - \\
\hline Average + STDEV & $33.8 \pm 5.6$ & $9.2 \% \pm 1.6 \%$ \\
\hline
\end{tabular}

\subsection{Result of Simulations}

\subsubsection{Collimated Source Simulations}

As described in the methodology paragraph, these simulations are reproducing the conditions in experimental setup. As reported in the first row of Table 2, the simulation, with the ideal conditions, has average front irradiance of $367.2 \mathrm{~W} / \mathrm{m}^{2}$ and rear of $46 \mathrm{~W} / \mathrm{m}^{2}$. As a result, the transmissivity is $12.5 \%$, almost $3 \%$ more than the experimental result. This overestimation of the transmissivity could be due to the perfect behaviour of the simulation if compared with experimental conditions. Unfortunately, in the experimental setup, a perfect perpendicular condition between solar simulator and receiver main axis cannot be not guaranteed. Moreover, the reflectivity of $53 \%$ is an average value of spectral reflectivity on flat surface reported in the literature. The surface of materials is quite rough ( $\mathrm{Ra}>3$ micron) this mean that a reduction of reflectivity can be observed [14]. Considering the ideal case and the worst case with reflectivity value of $30 \%$ and the nonperpendicular condition, average irradiances in the rear face were calculated. Therefore, decreasing $\alpha$ of $3^{\circ} \mathrm{Deg}$, the average rear irradiance reduces to $33.6 \mathrm{~W} / \mathrm{m}^{2}$ and $32.5 \mathrm{~W} / \mathrm{m}^{2}$ for $53 \%$ and $30 \%$ reflectivity, respectively; the transmissivity drops to $9.2 \%$ and $8.9 \%$, respectively. The average value of transmissivity considering a reflectivity of $53 \%$ is $11.3 \% \pm 1.5 \%$, see Table 2 . While the average amount of transmissivity considering a reflectivity of $30 \%$ is $10.9 \% \pm 1.5 \%$. Therefore, the experimental transmissivity $(9.2 \% \pm 1.6 \%)$ has a good overlap with simulated transmissivity. This result indicates that the simulation can produce robust results.

Table 2. MCRT simulations with collimated source varying the angle between radiation source and receiver main axis. Two different receiver reflectivity @ are considered $53 \%$ and $30 \%$.

\begin{tabular}{|c|c|c|c|c|c|}
\hline \multirow{2}{*}{$\begin{array}{l}\text { Source Conditions } \\
\text { Angle to Receiver } \\
\text { Main Axis (Deg) }\end{array}$} & \multirow{2}{*}{$\begin{array}{c}\text { Average } \\
\text { Irradiance Front } \\
\text { Surface } \mathrm{W} / \mathrm{m}^{2}\end{array}$} & \multicolumn{2}{|c|}{$\begin{array}{l}\text { Average Irradiance } \\
\text { Rear Surface }\left(\mathrm{W} / \mathrm{m}^{2}\right)\end{array}$} & \multicolumn{2}{|c|}{ Transmissivity $\%$} \\
\hline & & $\varrho=53 \%$ & $Q=30 \%$ & $Q=53 \%$ & $Q=30 \%$ \\
\hline $90^{\circ}$ & 367.2 & 46 & 45.9 & $12.5 \%$ & $12.5 \%$ \\
\hline $89^{\circ}$ & 365.9 & 44.2 & 43.4 & $12.1 \%$ & $11.9 \%$ \\
\hline $88^{\circ}$ & 365.7 & 37.9 & 39.1 & $10.4 \%$ & $10.7 \%$ \\
\hline $87^{\circ}$ & 364.6 & 33 & 32.5 & $9.2 \%$ & $8.9 \%$ \\
\hline Average & 365.9 & $41.5 \pm 5.7$ & $40.2 \pm 5.8$ & $11.3 \% \pm 1.5 \%$ & $10.9 \% \pm 1.6 \%$ \\
\hline
\end{tabular}

\subsubsection{Photon Number Sensitivity Analysis}

In the considered MCRT simulations, only a part of the photons reaches receiver geometries. Hence, the heat flux inside the receiver could depend on a number of photons generated by the solar source, which then evolve in the considered system. A sensitivity analysis of this flux inside the receiver was carried out with a generated photon number range between 25 and 200 million. In Figure 6 , the evolution of radiative power along the configuration 1 receiver main axis is reported and six different simulation results are compared. From the comparison, it appears clearly that the evolution of radiative power does not depend on the number of photons. The variation of impinging radiation power that enters in the receiver front face, varying the photons number between 25 and 200 million, is less than $0.005 \%$. Therefore, the number of 100 million photons was chosen as a compromise for the standard number for simulations. 


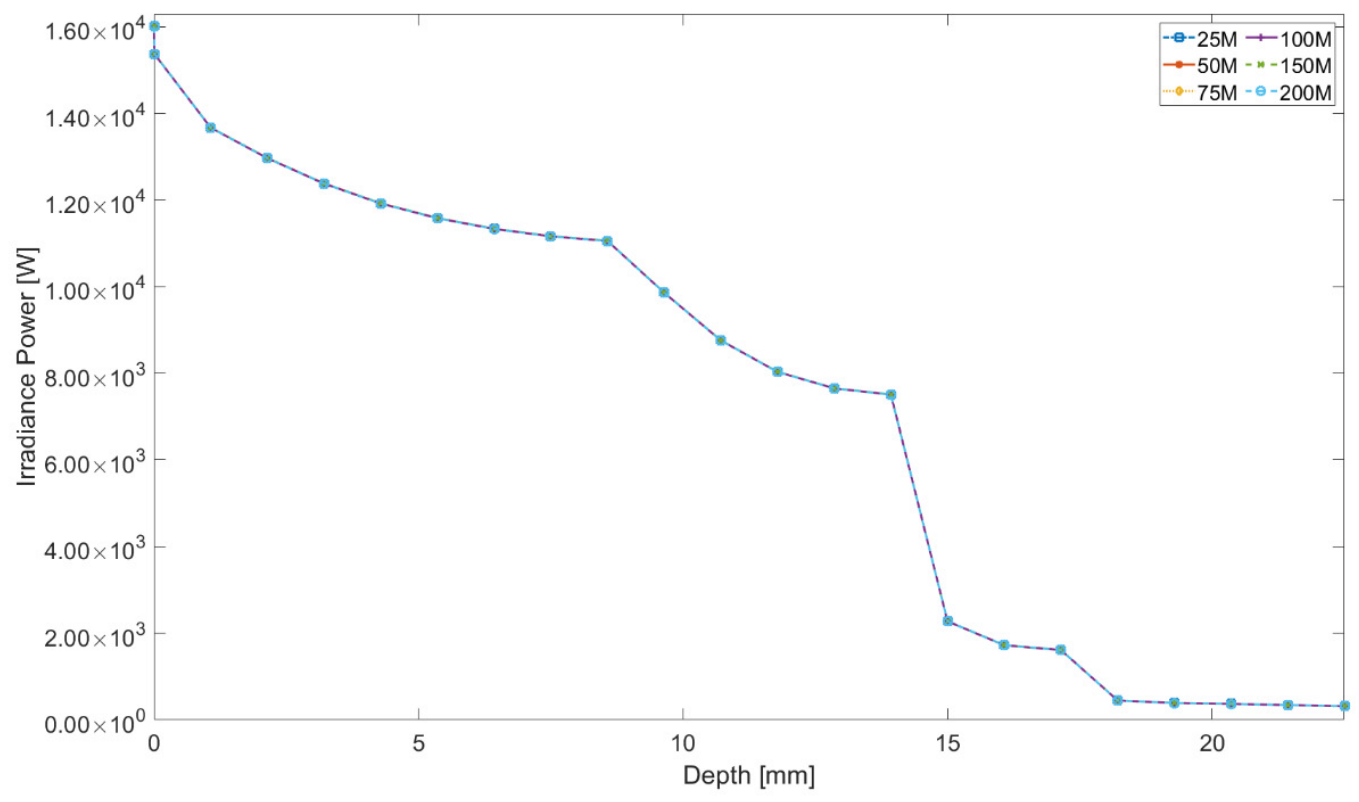

Figure 6. Irradiance power evolution on forward direction varying the number of photons: six different simulations from 25 to 200 million photons.

\subsubsection{MCRT Simulation Results with Concentrated Source}

In order to evaluate the evolution of radiation inside the HVR receiver, a complete postprocessing routine was developed. This post-processing routine analyses the irradiance on every auxiliary plane, including all the lateral and cutting planes of the configuration 1.

Figure 7 shows the relative radiative power evolution inside the HVR receiver in the configuration 1. All results are weighted to incoming power from the Contest solar dish that is 1.6015 $\times 10^{4} \mathrm{~W}$. This incoming radiation evolves in the forward direction from the frontal face to the rear decreasing to less $2 \%$ of the incoming power that leaves the HVR rear face. It may be noted that the forward power trend has four exponential decays. These decays depend on the different HVR layers that radiative power is hitting on the way to the rear face. The exponential decay gets slower in the last part of every layer due to lower presence of the solid body. As well as on forward direction, the evolution of backward power is influenced by solid geometry. This effect could be observed in the reversed decay of the backward direction power, especially from $15 \mathrm{~mm}$ to $10 \mathrm{~mm}$, where the second layer was absorbing or reflecting more than $5 \%$ of the total incoming power.

The power absorption also shows a trend that is influenced by the presence of solid material. On this parameter, the importance of the frontal surface of the third layer, at $15 \mathrm{~mm}$ from the frontal surface, that absorbs slightly less than $20 \%$ of the incoming power, should be noted.

The power spread on lateral non-solid surfaces through the whole absorber is $5.3 \%$ of the total incident power. Most of this power is dispersed in the first and second layer of absorber geometry. Only $0.1 \%$ of radiation spread on the lateral non-solid surface, therefore the first part of absorber makes the ongoing flux more collimated. 


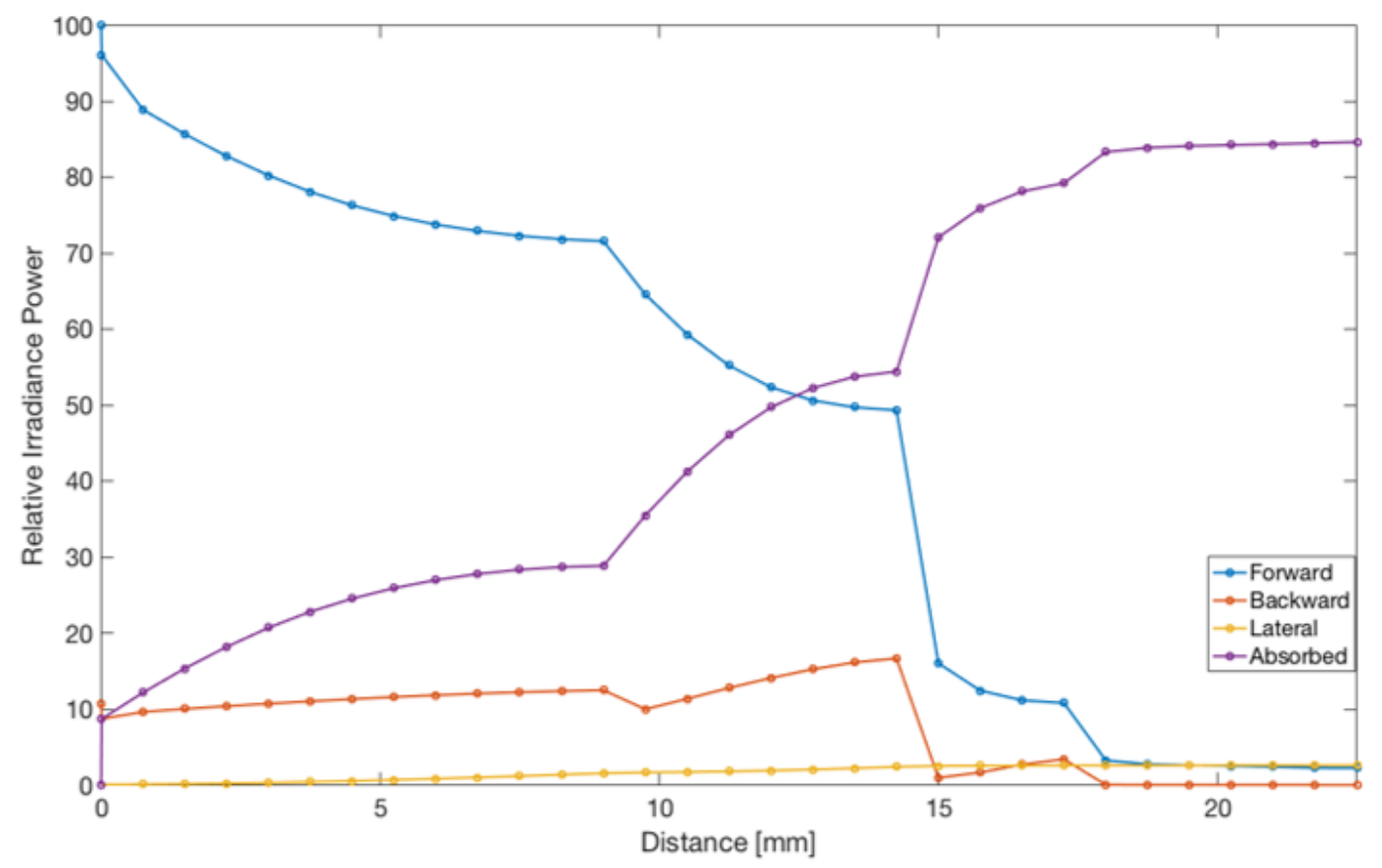

Figure 7. Relative radiative power evolution along main axis of configuration 1: radiative power moving along the forward direction (from front to rear absorber face), backward direction, lateral non-absorbed power, absorbed power.

Figure 8 reports a comparison of the power evolution along the main axis of the two configurations. Three different solutions are compared: configuration 1, configuration 2 and configuration 1 central. The configuration 1 central utilizes the results of the simulations of configuration 1 but only the volume of the configuration 2 is considered in post-processing of the results. It may be noted that there are some important differences of forward radiation power between configurations 1 and 2 in the first part of the absorber. The configuration 1 power decays more rapidly than the other configurations. At the end of layer 1 , the configuration 1 power is $2 \%$ lower than the configuration 2 power. If this comparison is moved to the configuration 1 central part only, this difference reduces to only $1 \%$. These differences are nullified in the receiver second part where the power difference decreases to $0.2 \%$. The different behaviour between the two configurations could be due to boundary conditions of configuration 2 . These conditions change the evolution of radiative power through non-solid lateral surfaces that, as explained earlier, have an important influence in the first and second layer. Despite the differences in radiation evolution, configuration 2 performs well in receiver losses with a variation from configuration 1 of $0.2 \%$ in both front and back losses. The configuration 2 has demonstrated a good overlap compared with configuration 1. This means the optical performance of volumetric receivers with this hierarchical structure has, as a feature, the scale invariance. We remark that this feature is very important to scale up at the industrial process HVR. 


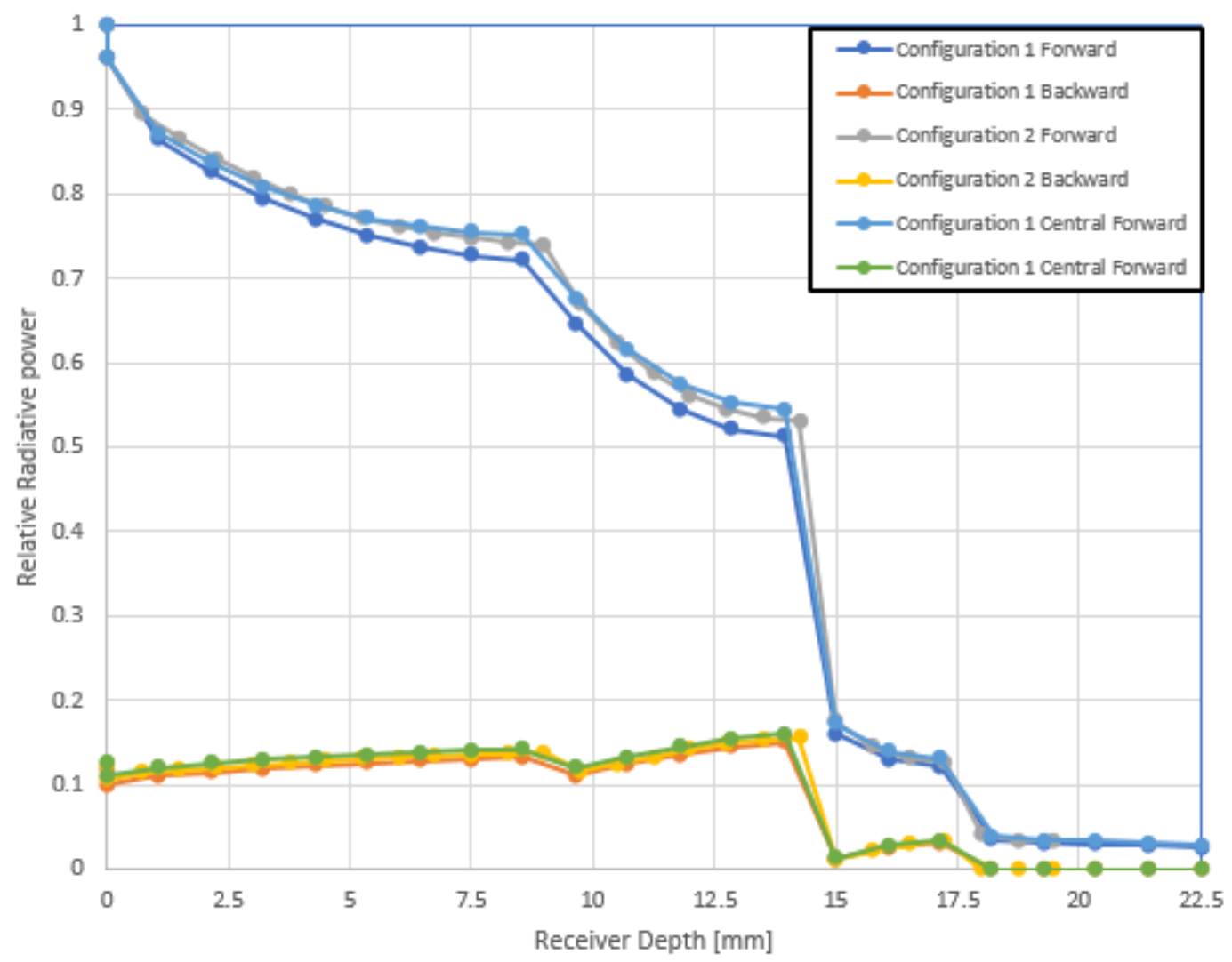

Figure 8. The relative radiative power attenuation along HVR main axis in both the considered configurations. The power is evaluated on the forward, from frontal face to the rear one, and backward direction.

\section{Conclusions}

In this work a detailed analysis of radiation propagation inside the hierarchical absorber was presented. This analysis includes both experimental measurements and numerical models. In the first part, using the experimental result, a model using MCRT simulations with a radiative collimated source was designed. In the second part, a MCRT simulations analyses HVR behaviour in two different configurations using a real concentrating mirror geometry. A comparison of results between the experimental protocol developed here and the MCRT simulation shows a good concordance indicating that the protocol is robust. The numerical simulation results have demonstrated a good concordance between the two HVR with different dimensions. This means that the use of a reduced receiver geometry allows a time and computational effort reduction that enables new prospects for the investigation of several geometries and conditions. A new experimental setup that could measure the power spread and reflected in the receiver geometry, is under development. The authors remark that this methodology can be used for other receiver geometries.

Author Contributions: L.P.: conduction of experimental analysis, conceptualization and perform of the MCRT simulations, data visualization, Writing, Editing and Review. R.B.: conduction of experimental analysis, data visualization, Writing, Editing and Review. L.C.: Supervision, Review \& Editing, Conceptualization. E.S.: Supervision, Review \& Editing, Conceptualization. All authors have read and agreed to the published version of the manuscript.

Acknowledgments: R.B. and L.C. give thanks for the support of the European Union's Horizon 2020 research and innovation program under grant agreement No. 731287 INSHIP project (Integrating National Research Agendas on Solar Heat for Industrial Processes).

Conflicts of Interest: The authors declare no conflict of interest. 


\section{Abbreviations}

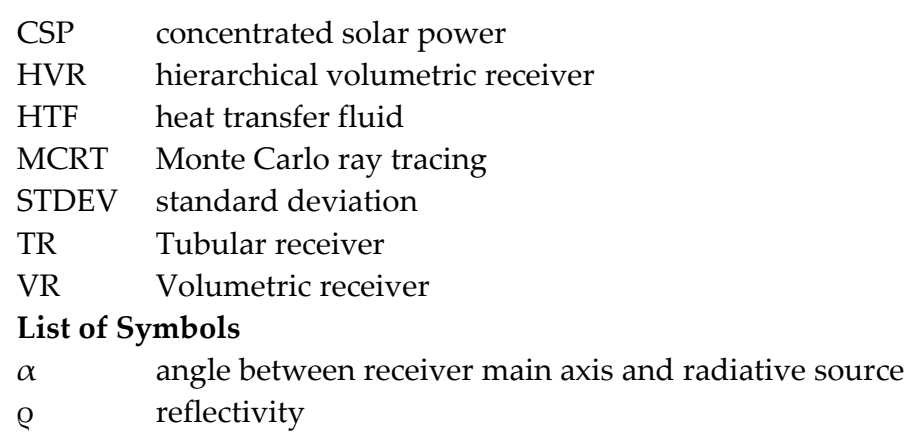

\section{References}

1. COP21. Available online: https://www.gouvernement.fr/en/cop21 (accessed on 2 April 2020).

2. United States Environmental Protection Agency. Sources of Greenhouse Gas Emissions. Available online: https://www.epa.gov/ghgemissions/sources-greenhouse-gas-emissions (accessed on 2 April 2020).

3. Avila-Marin, A.L.; Caliot, C.; Alvarez de Lara, M.; Fernandez-Reche, J.; Montes, M.J.; Martinez-Tarifa, A. Homogeneous equivalent model coupled with P1-approximation for dense wire meshes volumetric air receivers. Renew. Energy 2019, 135, 908-919, doi:10.1016/j.renene.2018.12.061.

4. Aichmayer, L.; Garrido, J.; Laumert, B. Thermo-mechanical solar receiver design and validation for a micro gas-turbine based solar dish system. Energy 2020, 196, 116929, doi:10.1016/j.energy.2020.116929.

5. Kalogirou, S.A. Solar Energy Engineering: Processes and Systems; Academic Press: Cambridge, MA, USA, 2013; ISBN 0-12-397256-6.

6. Gigantino, M.; Kiwic, D.; Steinfeld, A. Thermochemical energy storage via isothermal carbonationcalcination cycles of MgO-stabilized SrO in the range of 1000-1100 ${ }^{\circ} \mathrm{C}$. Sol. Energy 2019, 188, 720-729, doi:10.1016/j.solener.2019.06.046.

7. Romero, M.; Steinfeld, A. Concentrating solar thermal power and thermochemical fuels. Energy Environ. Sci. 2012, 5, 9234-9245, doi:10.1039/C2EE21275G.

8. Cagnoli, M.; Froio, A.; Savoldi, L.; Zanino, R. Multi-scale modular analysis of open volumetric receivers for central tower CSP systems. Sol. Energy 2019, 190, 195-211, doi:10.1016/j.solener.2019.07.076.

9. Ho, C.K.; Iverson, B.D. Review of high-temperature central receiver designs for concentrating solar power. Renew. Sustain. Energy Rev. 2014, 29, 835-846, doi:10.1016/j.rser.2013.08.099.

10. Avila-Marin, A.L.; Fernandez-Reche, J.; Martinez-Tarifa, A. Modelling strategies for porous structures as solar receivers in central receiver systems: A review. Renew. Sustain. Energy Rev. 2019, 111, 15-33, doi:10.1016/j.rser.2019.03.059.

11. Ávila-Marín, A.L. Volumetric receivers in Solar Thermal Power Plants with Central Receiver System technology: A review. Sol. Energy 2011, 85, 891-910, doi:10.1016/j.solener.2011.02.002.

12. Fend, T. High porosity materials as volumetric receivers for solar energetics. Opt. Appl. 2010, 40, $271-284$.

13. Kribus, A.; Gray, Y.; Grijnevich, M.; Mittelman, G.; Mey-Cloutier, S.; Caliot, C. The promise and challenge of solar volumetric absorbers. Sol. Energy 2014, 110, 463-481, doi:10.1016/j.solener.2014.09.035.

14. Luque, S.; Menéndez, G.; Roccabruna, M.; González-Aguilar, J.; Crema, L.; Romero, M. Exploiting volumetric effects in novel additively manufactured open solar receivers. Sol. Energy 2018, 174, 342-351, doi:10.1016/j.solener.2018.09.030.

15. Pitz-Paal, R.; Hoffschmidt, B.; Böhmer, M.; Becker, M. Experimental and numerical evaluation of the performance and flow stability of different types of open volumetric absorbers under non-homogeneous irradiation. Sol. Energy 1997, 60, 135-150, doi:10.1016/S0038-092X(97)00007-8.

16. Cagnoli, M.; Savoldi, L.; Zanino, R.; Zaversky, F. Coupled optical and CFD parametric analysis of an open volumetric air receiver of honeycomb type for central tower CSP plants. Sol. Energy 2017, 155, 523-536, doi:10.1016/j.solener.2017.06.038.

17. Nakakura, M.; Bellan, S.; Matsubara, K.; Kodama, T. Conjugate radiation-convection-conduction simulation of volumetric solar receivers with cut-back inlets. Sol. Energy 2018, 170, 606-617, doi:10.1016/j.solener.2018.06.006. 
18. Capuano, R.; Fend, T.; Stadler, H.; Hoffschmidt, B.; Pitz-Paal, R. Optimized volumetric solar receiver: Thermal performance prediction and experimental validation. Renew. Energy 2017, 114, 556-566, doi:10.1016/j.renene.2017.07.071.

19. Sedighi, M.; Padilla, R.V.; Alamdari, P.; Lake, M.; Rose, A.; Izadgoshasb, I.; Taylor, R.A. A novel hightemperature $\left(>700{ }^{\circ} \mathrm{C}\right)$, volumetric receiver with a packed bed of transparent and absorbing spheres. Appl. Energy 2020, 264, 114705, doi:10.1016/j.apenergy.2020.114705.

20. Alberti, F.; Santiago, S.; Roccabruna, M.; Luque, S.; Gonzalez-Aguilar, J.; Crema, L.; Romero, M. Numerical analysis of radiation propagation in innovative volumetric receivers based on selective laser melting techniques. AIP Conf. Proc. 2016, 1734, 030001, doi:10.1063/1.4949053.

21. Roccabruna, M.; Fabio, M.; Fabio, R.; Crema, L. Solar Flux Map Distribution of a Parabolic-Spheric Dish Based on Photographic Method. In Proceedings of the 12th International Conference on Solar Energy for Buildings and Industry (ISES EuroSun 2018), Rapperswil, Switzerland, 10-13 September 2018; doi:10.18086/eurosun2018.10.10.

22. Blanco, M.J.; Amieva, J.M.; Mancillas, A. The Tonatiuh Software Development Project: An Open Source Approach to the Simulation of Solar Concentrating Systems. In Proceedings of the ASME 2005 International Mechanical Engineering Congress and Exposition, Orlando, FL, USA, 5-11 November 2005; pp. 157-164; doi:10.1115/IMECE2005-81859.

23. Sun 2000 Class A. Available online: http://abet-technologies.com/solar-simulators/sun-2000-class-a/ (accessed on 28 May 2020).

24. RS PRO Solar Power Meter ISM400, Solar Power|RS Components. Available online: https://uk.rsonline.com/web/p/solar-power-meter/1232218/ (accessed on 28 May 2020).

(C) 2020 by the authors. Licensee MDPI, Basel, Switzerland. This article is an open access article distributed under the terms and conditions of the Creative Commons Attribution (CC BY) license (http://creativecommons.org/licenses/by/4.0/). 